\title{
Assessment, Pretreatment and Treatment of Pharmaceutical Production Wastewaters in the Roche Group
}

\author{
Jürg Oliver Straub*ab, Christoph Acklinc, Jürgen Klemmer ${ }^{d}$, Marco Aurélio Kurlbaume, Bin Lif, \\ Martin Studer ${ }^{c}$, Roland Zurbrügg ${ }^{c}$, and Andreas Häner*a
}

\begin{abstract}
The manufacturing of pharmaceuticals also produces wastes, mainly wastewaters (WWs). These WWs must be responsibly managed. Sometimes, the organic contents of these WWs are not easily removable in standard WW treatment, hence technical options must be investigated to pretreat such WWs in order to remove or destroy the recalcitrant compounds, mostly the active pharmaceutical ingredients themselves. This contribution from a pharmaceuticals company describes WW assessment and management principles, the search for pretreatment options and several case studies on WW (pre)treatment at some pharma production sites of the Roche Group.
\end{abstract}

Keywords: Assessment · Management · Pharmaceuticals · Pretreatment · Production $\cdot$ Treatment · Wastewater

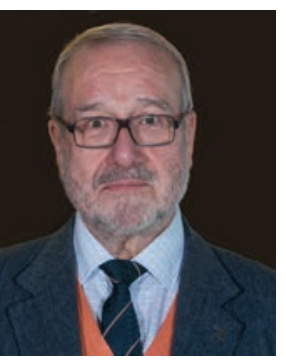

Jürg Oliver Straub, born 1954, is a Swiss biologist with a Dr phil from the University of Basle, Switzerland; he is a Chartered Biologist and a Fellow of the Royal Society of Biology (London, UK). Oliver originally started working in the Environmental Protection Department of F.HoffmannLa Roche Ltd in Basle in 1987. From 1998 to his retirement in 2019, he was the Environmental Risk Assessor in Roche Group SHE (Safety, Health \& Environment), where his main responsibility was regulatory environmental risk assessment for Roche pharmaceuticals. Oliver was also involved in investigating potential environmental problems from the production of pharmaceuticals and diagnostics at Roche, including excipients, intermediates, ancillary compounds and antibiotics. For the High Production Volume Chemicals programme, he produced comprehensive assessments of physico-chemical, human-health and environmental hazards and risks for Citric acid, Isophytol, Linalool and Pseudoionone, all of which were endorsed by the OECD. Oliver was a long-term member of the Swiss Experts' Commission for Ecotoxicology and of the European research pharma industry (EFPIA) Task Force on Pharmaceuticals in the Environment (PIE). He participated in research programmes on fate, effects and risks of PIE, e.g., ERAPharm, START, Strategie MicroPoll, NoMiracle, PHARMAS, TransRisk, iPiE and ChemPop. He is now an independent consultant.

\section{Introduction}

Pharmaceutical production causes wastes. Chemical synthesis, biotechnological manufacturing and galenical formulation result in contaminated wastewaters (WWs), beside organic liquid wastes, sometimes solid wastes and gaseous wastes. All these wastes need proper assessment, treatment and disposal.[1] Specifically, the 'Roche Position on Pharmaceuticals in the
Environment (PIE)' ${ }^{[2]}$ states that "Pharmaceuticals may enter the environment from production, from patient use and excretion or from improper disposal. While such releases are generally unintended, Roche basically believes that the presence of pharmaceuticals in the environment is undesirable and therefore should be minimised whenever feasible. Those releases that still occur must be assessed in a careful, scientific and differentiated way; if indicated such releases must be actively managed, treated and reduced." This brief communication gives an overview of WW management, a description of assessment and some case studies in pretreatment and treatment of WWs from the point of view of pharmaceutical industry, with examples from different kinds of pharmaceutical productions in the Roche Group.

\section{Wastewater Testing and Assessment}

\subsection{Initial Wastewater Testing and Assessment}

The Roche headquarters site in Basle, Switzerland was and still is the main development plant for chemical productions. Roche Basle started testing chemical synthesis WWs in the 1970s [H. Gröner, retired from the then Technical Safety and Environmental Protection Department, Roche Basle, pers. comm.] (Fig. 1). WW samples of all single production steps (generally from the 'kilolab', where a laboratory synthesis is ramped up to larger-scale industrial production) are assayed for removability of organic constituents in activated sludge (AS) treatment through biodegradation or adsorption, using a modified inherent Zahn-Wellens biodegradation test ${ }^{[3]}$ setup; more recent biotechnological production WWs are tested in the same way. Briefly, WW samples are analysed for total organic carbon (TOC) content, then mixed in 2-L Erlenmeyer flasks with AS (50\% from a mainly domestic and 50\% from an industrial WW treatment plant (WWTP)) to a final AS concentration of $200 \mathrm{mg}$ dry weight/L and incubated under constant aeration, with a magnet stirrer to prevent sedimentation, at room temperature. Subsamples from the flasks are taken on a regular basis, filtered and analysed

${ }^{*}$ Correspondence: Dr J. O. Straub, Dr A. Häner, E-mail: era.dr.j.o.straub@gmail.com, andreas.haener.ah1@roche.com

aF.Hoffmann-La Roche Ltd, Group SHE (LSR), CH-4070 Basle, Switzerland; 'bRA Competence, Carmenstrasse 14, CH-4123 Allschwil, Switzerland; ' $F$.Hoffmann-La Roche AG, Site SHE (MSU), CH-4070 Basle, Switzerland; 'Roche Diagnostics GmbH, Wastewater Treatment, Nonnenwald 2, D-82377 Penzberg, Germany; eProdutos Roche Químicos e Farmacêuticos S.A., Site SHE, Estrada dos Bandeirantes 2020, Jacarepaguá, Rio de Janeiro 22775-109, Brazil; fShanghai Roche Pharmaceuticals Ltd, Site SHE, 1100 Long Dong Avenue, Pudong, Shanghai 201203, People's Republic of China 
for dissolved organic carbon (DOC). The loss of DOC over time, compared with the initial TOC, gives a measure of removal (or not) of organic constituents. A minimum removal of $85 \%$ as required by the Swiss Water Protection Ordinance ${ }^{[4]}$ is required to pass this test. Other endpoints, e.g., decrease in biochemical oxygen demand (BOD) or chemical oxygen demand (COD) over time, may be examined as well. Also, other properties will fail the test, e.g., if the assay shows toxicity to AS microorganisms through disintegration of the AS flocs, or if there are heavy metals or certain solvents in the WW above defined limit concentrations, or if the $\mathrm{pH}$ is outside of the range 5-9.

Production WWs only get an internal discharge permit into the Roche Basle industrial WW if they have passed the test. Otherwise they must be either pretreated until they pass the test or, if that proves unfeasible, disposed of through combustion in a special waste incinerator. This WW assessment for all production steps forms an integral part of the production dossier; this means that also in case of outsourcing a production to third-party contract manufacturing operations those WWs originally assessed at Roche will be (pre)treated in an appropriate manner.

\subsection{Site-specific Evaluations, Environmental Risk Assessment}

Removability through physico-chemical pretreatment or in a WWTP relates to the specific properties of the substance(s) involved, yet removal will hardly ever be $0 \%$ or $100 \%$, but mostly somewhere in-between. However, this figure from a lab test does

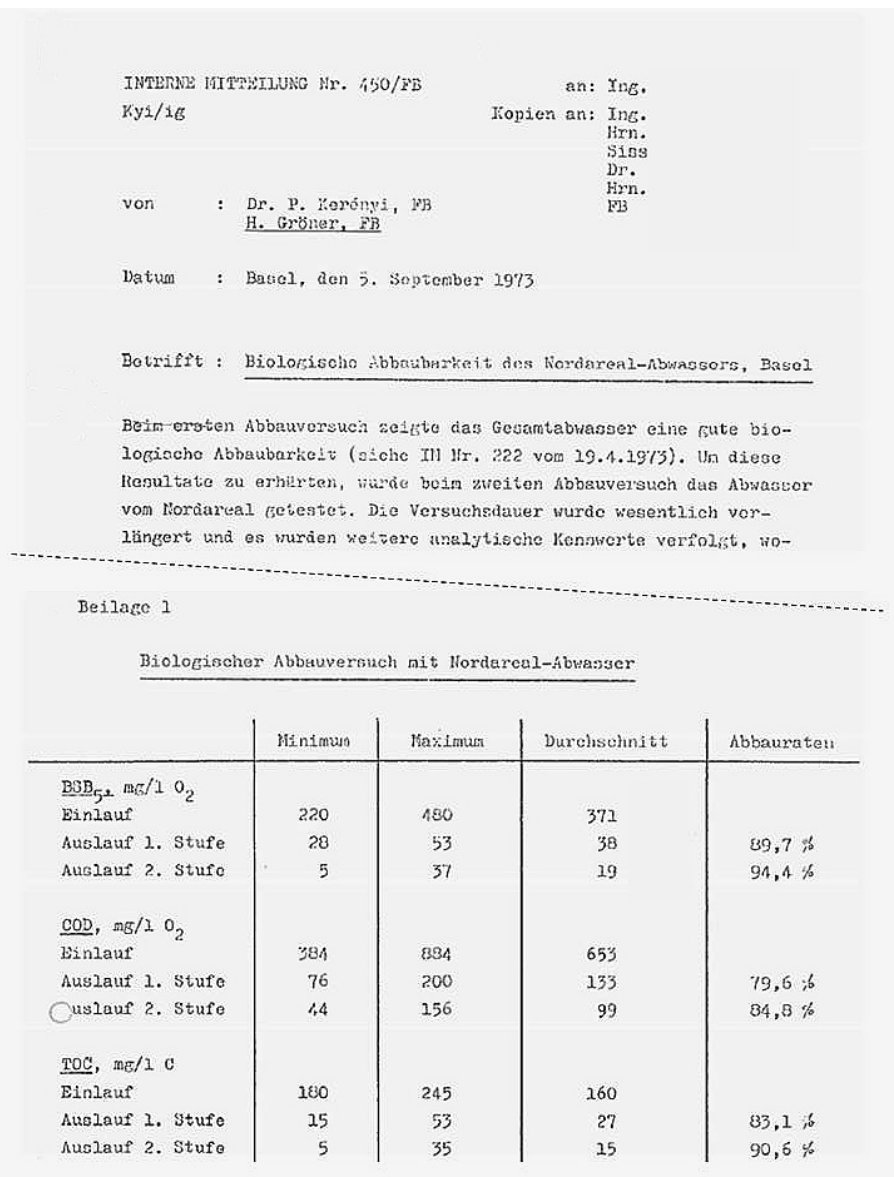

Fig. 1. One of the earliest internal wastewater assessments from Roche Basle dating to 1973 , showing the removability of the organic constituents as decrease in BSB $_{5}$ (German abbreviation for biochemical oxygen demand over 5 days), as decrease in COD (chemical oxygen demand), and as decrease in TOC (total organic carbon), all from start to end of the test, or from influent to effluent of the test set-up. Depending on the parameter applied, this wastewater reached $84.8-94.4 \%$ removal/degradability over 28 days. not refer to the specific conditions at a given production site, where the availability of an industrial or a municipal/mixed WWTP, with different substance concentrations, flow rates, adaptation of the AS, AS concentration, hydraulic and AS retention times, or possibly precipitation, flocculation, denitrification, dephosphatation, filtering or other additional treatment steps, may have a strong influence on removal rates. ${ }^{[5]}$ Also, the dilution factor of the receiving water and its characteristics as a freshwater or marine environment may be important for the further fate and potential risks of non-removed compounds. In such cases, knowledge about removability in lab tests alone is not sufficient to assess potential risks of discharged residues, but environmental risk assessment (ERA) methods must be adopted to estimate such risks.

An ERA consists of comparing a predicted environmental concentration (PEC) with a predicted no-effect concentration for environmental organisms (PNECenv) for a given environmental compartment. As WWs will pass through a WWTP to end up in receiving waters, initial PECs for surface waters are derived from the maximum amounts of a given substance entering a WW, removal during pretreatment or in a WWTP, and dilution in the receiving water. ${ }^{[6]}$ Such PECs may be refined using further data on environmental fate or may be complemented by measurement data. PNECenv values can be derived from defined minimum ecotoxicity datasets for different organisms, using assessment factors to account for the character of the datasets (number of species tested, acute or chronic data). ${ }^{[7]}$ An initial ERA can then be made by deriving a risk characterisation ratio (RCR), dividing PEC by PNECenv. RCRs $\geq 1$ denote potential risk while RCRs $<1$ suggest no immediate risk. Initial RCRs may be refined through incorporation of more precise data on amounts, additional information on pretreatment efficacy, the specific WWTP or on environmental fate, or on chronic ecotoxicity testing, but in all cases the specific local situation needs to be considered for a more definitive ERA.

\subsection{Special Investigations and Pretreatments}

In certain cases, particularly from formulation (see Section 3.3), single recalcitrant or potentially ecotoxic WWs are investigated in more detail for the possibility of physico-chemical pretreatment. In order to ensure the destruction or removal of highly active pharmaceutical ingredients (APIs), such investigations may encompass physical removal through precipitation, flocculation, or adsorption to activated charcoal or other substrates, possibly furthering hydrolysis through raising or lowering the $\mathrm{pH}$, with or without additionally heating the WW, or ozonation. Additionally, treatment with UV radiation, or advanced oxidation processes (AOPs) using UV with photosensitisers or oxidisers, may be tested in a specialised contract research laboratory.

\section{Case Studies}

The following case studies illustrate the principles of managing pharmaceutical production WWs through assessing, refining the ERA, pretreating, treating in a WWTP, or - ultimately - incineration.

\subsection{Chemical Production}

\subsubsection{Chemical Production Wastewaters at Roche Basle}

WW assessment and (pre)treatment have been described above (Sections 2.1-2.3). Roche Basle sends its chemical research and development (R\&D) and production WWs to the industrial WWTP Pro Rheno in Basle. However, WW management goes beyond assessment and internal discharge permit. Autosamplers are installed in every R\&D and production building that collect and store time-proportional WW samples, to be analysed in case of problems. The total chemical WW collects in a large pump well, from which the mixed WW is pumped through a tunnel of approximately $3 \mathrm{~km}$ length to the WWTP; 
in view of relatively low WW volumes this takes a couple of hours. A sample is continuously diverted from the pump sump to an in-house automated WW Monitoring Station, where $\mathrm{pH}$, conductivity, solvents, metals, TOC, volatile organohalogens and bacterial toxicity are measured. If any of those sentinel devices registers a exceedance of a limit value, an alarm allows to separate the corresponding Roche WW stream in a retention basin at the ProRheno WWTP, thanks to the time the WW needs to get there; it can than be assessed and treated separately. Beside the online monitoring, the WW sample in the WW Monitoring Station is also fed into a pilot WWTP (Fig. 2), with a mixing and volume buffering tank (as normally there is no production over weekends but a WWTP needs to run 7/7 d), $\mathrm{pH}$ neutralisation, an aerobic biodegradation tank with the same AS concentration and hydraulic residence time as in the big WWTP, and a settling tank for the AS. Automated DOC measuring of the effluent of the pilot WWTP, compared with time-shift-corrected TOC measurement of the influent, allows for a continuous determination of the total Roche WW biodegradability or removability. This would not be possible in the big WWTP as the WWs from several companies are mixed before treatment; here only an overall degradability/ removability can be reported. However, comparison of removal rates in the Roche pilot plant with removal rates determined at Pro Rheno WWTP over $>30$ years suggest that the big WWTP has highly comparable or even slightly better degradation rates than the Roche Monitoring Station, which may reflect a greater temporal stability, or a greater degradation potency, in view of a much larger AS volume on one hand and additional, different WW influxes from third parties on the other. Hence, the removability determined in the Roche Monitoring Station is taken as representative for the large WWTP.

\subsection{Biotechnological Production}

\subsubsection{Biotechnological Production Wastewaters at Roche Penzberg}

Roche Penzberg in Germany is a large production and research site for diagnostic active substances and APIs, most of which are produced by transfected cells or micro-organisms grown in special media. Like other research pharma and diagnostics companies, Roche has more and more biotechnological productions of monoclonal antibodies, beside other 'biologics', viz., peptide hormones, enzymes or other functional proteins for diagnostics products. Proof-of-concept evidence shows that such biologics themselves are not expected to cause any environmental problems, as shown by the ready biodegradability and the low ecotoxicity of a set of biologics tested on behalf of Roche. ${ }^{[8]}$ Some biotechno-

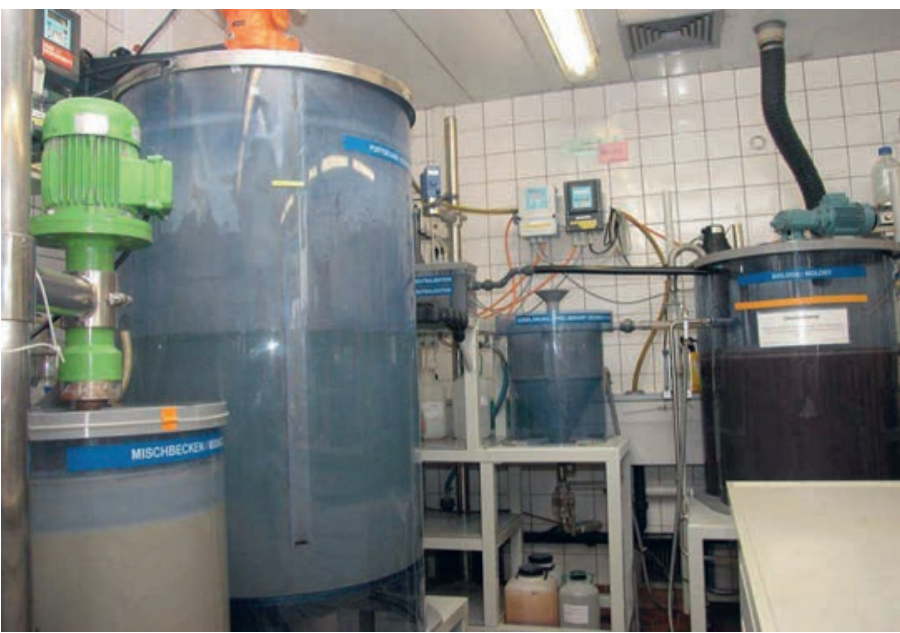

Fig. 2. Pilot WWTP in the Roche Basle WW Monitoring Station. Photo CRoche Basle. logical production WWs have been assessed at the Penzberg and Basle sites, and have also been found to cause no risks. ${ }^{[9]}$

In view of steadily increasing productions at Penzberg over the past 20 years, WW management and the on-site industrial WWTP (Fig. 3) have been continuously adapted and upgraded to deal with the amounts and qualities of the different WW streams. ${ }^{[10]}$ WW testing and assessment as outlined above assigns single WW streams to one of four classes.

1) General WWs are treated in five aerobic reactors in series with an AS concentration of 6.5-7.5 g dry weight/L, bubble aeration and a hydraulic retention time in the biodegradation basins of $\sim 28 \mathrm{~h}$. After this step, the WWs are filtered in a membrane unit, analysed on-line for regulatory parameters and finally discharged into the local River Loisach.

2) Concentrated, high organic load WWs without any constituents inhibitory to anaerobic bacteria are first treated in two large anaerobic digester towers. The installation of the first anaerobic digester nearly 10 years ago, after extensive appropriate tests, resulted in sufficient biogas (methane) production for a co-generation plant to satisfy the electricity demand of the whole WWTP and to deliver steam into the plant process steam network

3) The effluent of the anaerobic digesters is treated in a closed reactor fed with pure oxygen and similar operating conditions as above (1) for a first aerobic biodegradation and nitrification, before being pumped to (1) for a second aerobic treatment.

4) Those few WWs that do not fulfill the requirements for any of the above classes are incinerated.

A refined WW management system like Penzberg's not only requires the treatment options (1), (2), (3) and (4), but also facilities to physically separate different WW streams from the source into at least three different categories, (1), (2), and (4), plus possibly (3) for specific aerobically degradable WWs that need an oxygen-fed reactor. Hence, prospective WW management activities become more and more important, from testing and assessing to allocating single WWs to categories (1) to (4), separating them at the source and organising different WW streams with changing volumes over time in storage tanks, in order to maintain the flow rate through the biological treatments (1) to (3) within workable limits. WW management also needs to link into production planning, beside storage tanks, instrumentation and control, to make optimal use of the technical facilities. Surplus AS from the Penzberg WWTP is digested and incinerated, assuring that any sorbed compounds do not reach the environment. This is important insofar as in many countries surplus AS is used as an agricultural fertiliser.

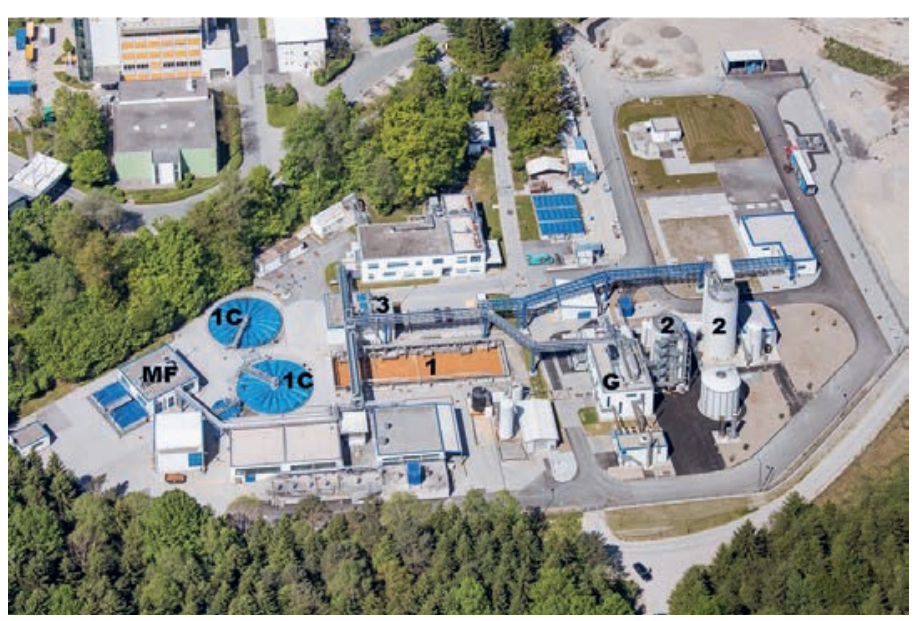

Fig. 3. Aerial view of the Roche Penzberg WWTP. (1) Serial open air-fed aerobic biodegradation tanks, (1C) covered air-fed aerobic biodegradation tanks. (2) Anaerobic digester towers. (3) Closed, pure-oxygen-fed biodegradation. $\mathrm{G}$ = Biogas electricity/steam co-generation. $\mathrm{MF}=$ Membrane filtration for activated sludge. Photo @Roche Penzberg. 


\subsubsection{Biotechnological Production Wastewaters at Roche Basle}

WW testing and assessment has determined no risk to the River Rhine for the growing biotechnological production at Roche Basle, but a constant increase of non-biodegradable organic buffers. These have been shown to pose no significant environmental risk, ${ }^{[9]}$ yet the regulatory Effluent Permit for Roche Basle restricts the discharge of recalcitrant organics in the treated WW to a fixed upper limit per week. Technical measures were sought to remove the buffers in a pretreatment in order to avoid constraining the local biotechnological production. The Basle team of biotechnological-production, engineering, biodegradation and environmental-assessment experts came up with the solution that (a) not all biotechnological WWs but only certain among them need pretreatment, e.g., those WWs that contain high concentrations of the above buffers, and that (b) nanofiltration excluding molecular masses above a given threshold should be able to produce an unproblematic pretreated WW (the permeate), while the retentate would be a concentrate of significantly smaller volume compared with the original WW, that would be sent for incineration. A pilot unit was set up to confirm that the technical requirements would be fulfilled and subsequently, a full-size installation (Fig. 4) was purpose-built. This installation has now been tested and, together with the corresponding WW management ensuring that only those WWs in need of pretreatment are sent through the filter unit, is working to full satisfaction. A detailed description of the installation, its operating parameters and performance will be published by Zurbrügg and colleagues (in preparation). The permeate is treated in the industrial WWTP in Basle, where all surplus AS is digested and incinerated.

\subsection{Galenical Formulation of Pharmaceuticals}

Formulation of pharmaceuticals combines the APIs with excipients for various purposes, from Adhesives over Antimicrobial Preservatives, Bulking Agents and many others, to Wetting/ Solubilising Agents, [11] into solid or liquid dosage forms. After formulation, the technical installations are cleaned, first by rinsing with water creating WWs, then using other appropriate means, and sterilised for the next production batch. Formulation was assessed in depth for the APIs ${ }^{[12]}$ and excipients ${ }^{[13]}$ for Roche Basle and also on local levels (not published), with some examples given in the following paragraphs.

\subsubsection{Formulation of Non-Biodegradable, High-Potency Active Pharmaceutical Ingredients}

i) A recalcitrant high-potency API was to be formulated at a Roche site in the People's Republic of China. A worst-case ERA

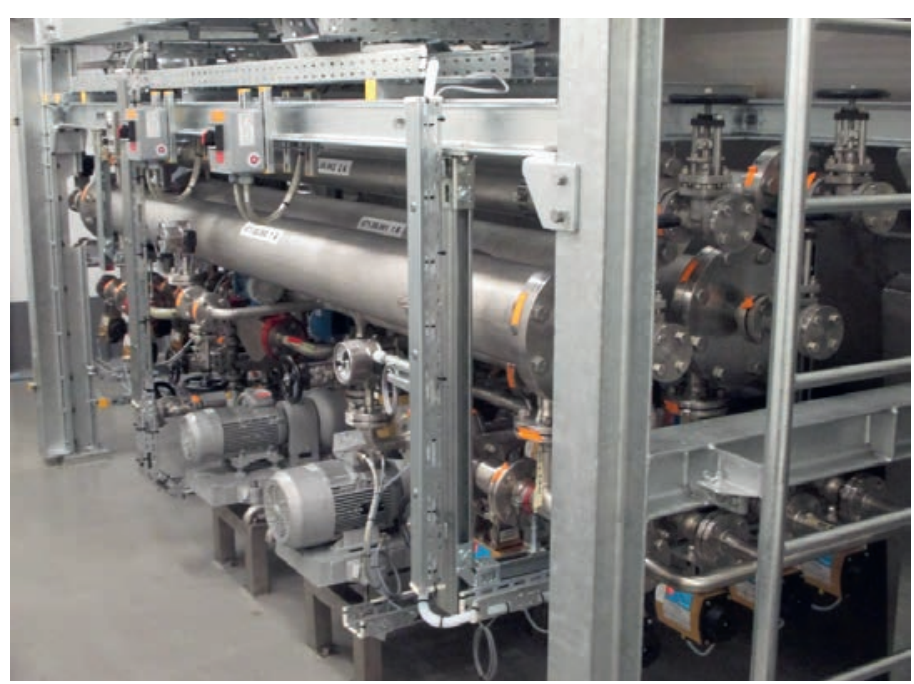

Fig. 4. Nanofiltration unit for selected biotechnology WWs at Roche Basle. Photo@Roche Basle. showed that the API in the effluent would pose no risk to the local receiving water, which has a very high dilution factor, but in view of the particularly high potency of this API and also of the likelihood of installing additional productions at other sites, an efficient pretreatment was sought. Tests were performed at Roche Basle with five different types of commercial activated charcoal, which showed that an inordinate amount of activated charcoal would be necessary to remove the API. In a second investigation at a contract research organisation, comparative AOP removal assays of the pure API, with different API and hydrogen peroxide concentrations, in tap water, under low- or middle-pressure mercury UV lamps, were set up for varying durations. These resulted in optimised process parameters for an AOP pretreatment combining UV light and $\mathrm{H}_{2} \mathrm{O}_{2}$ with temperature and $\mathrm{pH}$ control for a predetermined duration, with the API being hydrolysed to a transformation product that has been shown to be biodegradable in a WWTP. ${ }^{[14]}$ The formulating site was asked to pretreat the WW by $\mathrm{UV} / \mathrm{H}_{2} \mathrm{O}_{2}$ under specified conditions for the required duration, then neutralise and drain to the factory WW; also, in order to confirm the destruction efficiency, to take samples before and after the pretreatment and determine the rate of API decrease. The pretreated WW is mixed with the other WWs from the plant and treated in an own on-site sequencing batch reactor WWTP (with surplus AS being incinerated), before being sent to a large mixed municipal/industrial WWTP and ultimately discharged to the Yangtze River with a very high dilution factor.

ii) A different recalcitrant API was to be formulated at a Roche site in Mexico discharging the WWTP effluent into a small river. Adsorption and hydrolysis tests at Roche Basle of the process WW showed insufficient removal of the API. Incineration was not feasible in this location, hence other methods for the destruction of the API were investigated by a contract institute on behalf of Roche. Initial AOP parameters were developed in pure water for a far-reaching destruction. However, in the actual WW the AOP was not satisfactory from an efficacy point of view, ${ }^{[15]}$ therefore further tests with ozonation were initiated, which eventually showed high removal at the average API concentration in the WW under specific conditions (Fig. 5A). ${ }^{[16]}$ This ozone-pretreated WW was tested again in the Roche modified Zahn-Wellens test, where it showed clearly improved biodegradability and no overt toxicity to AS microorganisms. Therefore, a dedicated flow-through pressure ozonation reactor was built (Fig. 5B) and installed at the plant, delivering good results with on average $>99.8 \%$ API destruction (average of 11 on-site measurements of API concentration before and after the reactor).

\subsubsection{Formulation of Antibiotics}

i) Tablets and liquid syrup containing a combination of two antibiotics are formulated in a Roche site in Brazil. (The two antibiotics themselves are synthesised by two third-party chemical manufacturing companies, which were both audited on-site and assessed as satisfactory regarding environmental and WW aspects by the first author together with one of Roche's professional SHE auditors in 2017.) For tablet formulation, predetermined amounts of both antibiotics and the necessary excipients are slightly humidified and mechanically mixed, then the mixture is transferred to a tabletting machine; the solid residues (a thin cake in the mixer, any material remaining in the tablet press) are flushed to the WW. For the liquid dosage form the mixing procedure is analogous to above, but uses more sterile water and different excipients to form a stable solution; again, any residues in the mixer are flushed to the WW. The formulating plant has an own industrial WWTP with a high AS concentration of $\sim 7 \mathrm{~g}$ dry weight/L and a very long total hydraulic residence time of just over $100 \mathrm{~h}$, of which $55 \mathrm{~h}$ in the aerobic AS basin. The effluent of the WWTP is discharged into a relatively small local river. 

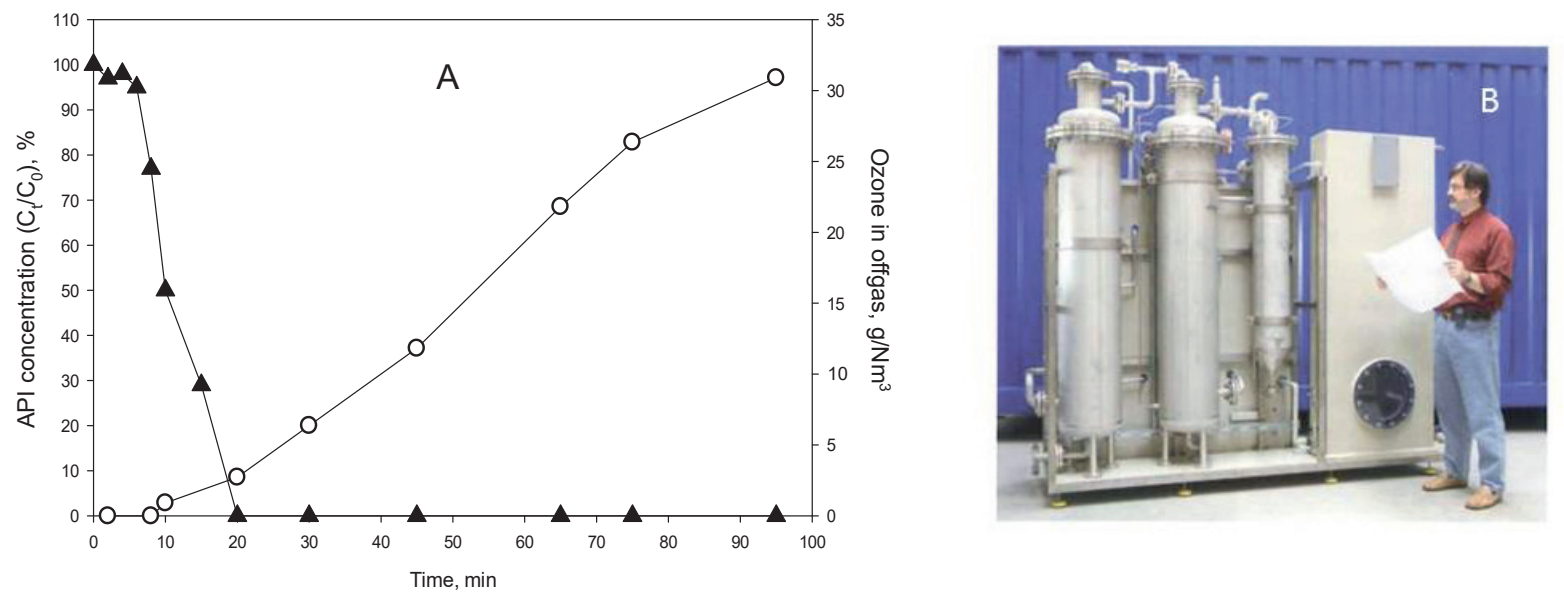

Fig. 5. A: Ozonation experiment of an API in water. Graph based on empirical data by Türk and Haep. ${ }^{[15]}$ Black triangles: API concentration normalised to starting concentration. White circles: Ozone concentration in off-gas. B: Bespoke flow-through ozonation reactor, built by EnviroChemie, Germany, on behalf of Roche.

Material balances for recent production runs showed an average loss rate of $0.7 \%$ for both solid and solution productions. Separate ERAs for both antibiotics were derived from the receiving water PEC, without considering any potential removal in the WWTP, and chronic-based PNECenv values were taken from literature. ${ }^{[17]}$ In addition, in view of the potential of antibiotic resistance formation or maintenance in surface waters, the receiving water PECs were divided by a PNEC based on bacterial minimal inhibition concentrations (PNECmic) as proposed by BengtssonPalme and Larsson in 2016. ${ }^{[18]}$ The RCRs showed potential risk for both antibiotics, for both PNECenv and PNECmic, indicating that either the ERA must be refined or that risk management measures must be taken.

Separating the first rinse from the mixers is technically not possible in this particular plant, due to closed production systems with integrated In-Process Cleaning (IPC). Hence, the site was asked to sample and analyse the concentration of both antibiotics during a worst-case parallel production run for solid and liquid antibiotic formulations, both in the WWTP influent mixing tank and, with due time shift, in the effluent of the WWTP, over $24 \mathrm{~h}$ each. Comparing the area under these curves showed $>98 \%$ removal for the first antibiotic and $>96 \%$ removal for the second, both of which are known to be degradable in a WWTP with adapted AS and long hydraulic residence time. ${ }^{[19,20]}$ These measured removal data allowed to refine the initial risk assessment for both PNECenv and PNECmic in the receiving water, resulting in no risk for both antibiotics. Hence, no additional risk management measures are necessary in this case. Surplus AS from this WWTP is binned and incinerated.

ii) A different antibiotic is formulated at a Roche site in the People's Republic of China. This antibiotic had been shown to be reliably destroyed through hydrolysis by increasing the $\mathrm{pH}$ for a specified time, so that the antibiotic lost its function, which is important in view of the spread and risks of antibiotic resistance. While a first ERA showed no risk for both PECenv and PECmic in the receiving water, the pretreatment procedure was set up as a standard for this kind of production, in view of parallel productions at other companies (see below), where WW treatment and local dilution would differ. The site started treating all WWs from this production, i.e. not just the first rinse, at the high $\mathrm{pH}$ for the required duration, then to neutralise and drain the pretreated batch to the plant WW. Repeat samplings and analyses, to determine the concentration of the API before and after the pretreatment, consistently showed $\geq 99 \%$ destruction. Repeating the ERA with the total residual API concentration confirms that the pretreatment works well and that the concentration of any residues reaching the receiving water is well below PNECenv and PNECmic, hence there is no cause for concern. The on-site and municipal/industrial WWTP as well as the AS incineration have been described above.

The same antibiotic is also formulated on behalf of Roche by a contract manufacturing organisation (CMO) in another province of the People's Republic of China. The production WW of the CMO is pretreated as described above, before it is discharged into a small on-site WWTP followed by a large mixed municipal/industrial WWTP. An initial ERA in this case highlighted potential risk for antibiotics resistance, due to no measurements being available regarding the efficiency of the pretreatment in that specific plant and also due to the application of an assumed default dilution factor in the receiving water, as no pertinent data were available in a Western language. The contractor was asked to determine the antibiotic concentrations before and after the pretreatment and to get information from the regional Water Board on the realistic local dilution factor. Measured pretreatment efficiency proved to be $>98 \%$ to $>99 \%$, comparable to above, and the actual dilution factor of 48 [pers. comm. from the contractor] is nearly five times higher than the originally assumed default value of 10 . Combining both new data in the updated ERA results in no evident risk for PNECenv and PNECmic, meaning no risk for the receiving water. For this WWTP, no information is available regarding surplus AS incineration or not, but in view of the irreversible inactivation of the antibiotic during the pretreatment, no risk from potential AS spreading on arable land is expected.

\section{Discussion}

WW management in the pharmaceutical industry is not a static target to be reached once, but rather a process that needs be started, built up and improved over time. It is not only the WW (pre)treatment technologies that change, but also the productions (when new APIs advance to the market or old APIs are not produced any longer), the overall volume of pharmaceutical WW, and the requirements for $\mathrm{WW}$, effluent or receiving water quality changing over time, in terms of discharge or effluent permits, official water quality criteria or standards. To cope with building up a WW system with all these variable boundary conditions and targets a sound WW management strategy is needed, such as has been outlined by Caldwell et al.[21] with the concept of a 'WW Maturity Ladder'.

Treatment, and where indicated, pretreatment, of pharmaceutical production WWs is part of the overall WW management, which begins with an initial assessment of the removability of the organic constituents, or specifically of the API(s), in a single process WW, as well as of potential toxicity or of the presence of heavy metals. However, in general this initial evaluation is not 
sufficient (except in case of readily degradable, nonproblematic WWs) but should be extended to an ERA factoring in local conditions, projected, worst-case or measured losses of the API to WW, technical data for the WWTP in question and the actual dilution in the receiving water. This allows for a site-specific assessment that gives a useful estimate of local PECs. To determine the potential risks, additionally a PNECenv is needed, based on chronic (for more recent APIs) or acute (for some older, 'legacy' APIs, or for new ones in development) ecotoxicity data. Such information may be found in the open scientific literature, in proposed or definitive Environmental Quality Standards of the EU Water Framework Directive, ${ }^{[e . g . g .22]}$ in industry publications like Vestel et al., ${ }^{[23]}$ or the Water and Environmental Technology Center pharmaceuticals PNEC compilation, ${ }^{[24]}$ on the Swedish FASS website, ${ }^{[25]}$ sometimes on company safety data sheets ${ }^{[26,27]}$ or on abbreviated ERAs for APIs published on the websites of some pharma companies. [28-30]

If indicated by the ERA or possibly by specific substance properties, e.g., highly potent APIs or antibiotics, pretreatment of WWs should be investigated. Various physical or physico-chemical procedures may be tested, from simple heating or $\mathrm{pH}$ shifts over ad- or absorption using activated charcoal or other substrates, nanofiltration, enhanced oxidation using different means, to several kinds of AOP, while incineration of the whole WW is normally the last resort. An ample body of literature on physico-chemical pretreatment options is available, as a short search on Google Scholar will confirm. In addition, simple to sophisticated tests and competent contract research organisations may help in finding the optimal solution for a given problem. In case pretreatment is not necessary because the local WWTP can be shown to reliably remove the substance in question, adsorption to AS as a possible removal pathway and the handling of surplus AS should be considered.

However, one should be conscious that any kind of pretreatment will generate costs, including environmental costs, from investments made, over increased energy consumption, additional raw materials needed, more $\mathrm{CO}_{2}$ produced, or other kinds of wastes generated. WW incineration in general is the last option, as an inordinate amount of energy is needed to evaporate water, often constituting well over $98 \%$ of a WW, to eventually combust the minor residues of recalcitrant or (eco)toxic organics. Therefore, a careful comparison between available pretreatment options should be made, to identify the optimal under the given circumstances.

Coming back to the first sentence of this contribution, "Pharmaceutical production causes wastes", specifically WWs. As outlined above, there are methods to assess and techniques to (pre)treat and thereby remove, inactivate or reduce such wastes, in particular organics of concern in the WWs. Concepts and methods have been developed to reduce all kinds of wastes (and risks) through 'Green Chemistry', as pioneered by Anastas and colleagues. [e.g., 31] Syntheses, reactants, solvents, catalysts, processes and process controls can be selected, amended and optimised, in order to produce under improved chemical and occupational safety conditions, with higher yield, with more recyclable byproducts and with a reduced total of losses, of nonrecyclable or ecotoxic wastes. However, while Green Chemistry is widely appreciated and also applied in the pharmaceutical industry, ${ }^{[e . g .,}{ }^{32-34]}$ no chemical reaction achieves $100 \%$ yield. Farreaching reduction is achievable, $[$ e.g., 32,35$]$ but zero waste production is illusory insofar as other wastes (spent absorbents, used filters, other solid wastes) or $\mathrm{CO}_{2}$ may be produced, or additional energy utilised, the generation of which creates wastes as well. Hence, while we can (and should) minimise wastes and WWs through the adoption of Green Chemistry principles and intelligent process optimisation, some residual wastes and WWs will always remain, and these need to be treated. In this sense, the application of sound waste and WW management, assessment methods, pretreatment and treatment techniques, as end-of-pipe solutions, are ultimately a consequent continuation of responsible pharmaceutical production.

\section{Acknowledgements}

Many thanks to former and current colleagues in the Roche Basle WW lab and in the Roche Group all over the world for taking responsibility for their WWs, supporting requests for data and in many cases helping by taking samples or getting additional information. Two postgraduate temps, C. C. Hoerger and K. C. Wirz, are acknowledged for their highly useful investigations of APIs and excipients in galenical production at Roche Basle. Without all these contributions over many years the current state of WW (pre)treatment at Roche Group sites would not have been attained.

\section{Declaration of Conflicts}

All authors are or used to be full-time employees of F.Hoffmann-La Roche Ltd and its affiliates, working in Roche site or Group environmental protection departments.

Received: September 2, 2019

[1] 'Roche Guidelines for the Assurance of Safety, Security, Health and Environmental Protection', F.Hoffmann-La Roche Ltd, Basle, Switzerland, 2019, pp. 36 ff, cited July 2, 2019, available from www.roche.com/dam/ jcr:5b95a6fa-fla8-4c00-9219-86e5b8d70e82/en/she-guidelines.pdf

[2] 'Roche Position on Pharmaceuticals in the Environment (PIE)', F.HoffmannLa Roche Ltd, Basle, Switzerland, 2019, cited July 2, 2019, available from www.roche.com/pharmaceuticals_in_the_environment.pdf

[3] R. Zahn, H. Wellens, Z. Wasser Abwasser Forsch. 1980, 13, 1.

[4] Swiss Federal Council, 'Waters Protection Ordinance' (WPO), SR 814.201 of 28 October 1998, status as of 1 June 2018, cited July 2, 2019, available from www.admin.ch/opc/en/classified-compilation/19983281/index.html

[5] H. Blok, 'A Quest for the Right Order. Biodegradation rates in the Scope of Environmental Risk Assessment of Chemicals', PhD Thesis, Utrecht University, The Netherlands, 2001, ISBN 90-74718-07-8.

[6] ECHA, 'Guidance on information requirements and Chemical Safety Assessment. Chapter R.16: Environmental exposure assessment', v 3.0, February 2016, European Chemicals Agency, Helsinki, Finland, 2016, cited July 3, 2019, available from echa.europa.eu/documents/10162/13632/information_requirements_r16_en.pdf

[7] ECHA, 'Guidance on information requirements and chemical safety assessment'. Chapter R.10: 'Characterisation of dose [concentration]-response for environment', May 2008, European Chemicals Agency, Helsinki, Finland, 2016, cited July 3, 2019, available from echa.europa.eu/documents/10162/13632/information_requirements_r10_en.pdf/bb902be7a503-4ab7-9036-d866b8ddce69

[8] J. O. Straub, 'Protein and Peptide Therapeuticals: An Example of «Benign by Nature» Active Pharmaceutical Ingredients', in 'Green and Sustainable Pharmacy', Eds. K. Kümmerer, K. Hempel, Springer, Berlin, 2010, pp. 127133.

[9] J.O. Straub, D. Gysel, U. Kastl, J. Klemmer, M. Sonderegger, M. Studer, Environ Toxicol Chem 2012, 3, 681 .

[10] J. Klemmer, CIT Plus 2016, 5, 30.

[11] 'United States Pharmacopeia, USP and NF Excipients, Listed by Functional Category', United States Pharmacopeial Convention, Baltimore MD, 2019, subscription online access database, cited July 4, 2019, available from $w w w$. usp.org/

[12] C. C. Hoerger, B. Dörr, C. Schlienger, J. O. Straub, Integr. Environ. Assess. Manag. 2009, 5, 331,

[13] K. C. Wirz, M. Studer, J. O. Straub, Sust. Chem. Pharm. 2015, 2, 28.

[14] R. Zurbrügg, Internal Report, F.Hoffmann-La Roche Ltd, Basle, Switzerland, 2014, unpublished.

[15] J. Türk, S. Haep, 'Forschungsbericht Nr. UWA 07/010', IUTA, Duisburg, Germany, 2007, on behalf of F.Hoffmann-La Roche AG, unpublished.

[16] E. Billenkamp, J. Straub, M. Studer, J. Türk, Pharma 2011, 1, 2.

[17] J. Tell, D. J. Caldwell, A. Häner, J. Hellstern, B. Hoeger, R. Journel, F. Mastrocco, J. J. Ryan, J. Snape, J. O. Straub, J. Vestel, Integr. Environ. Assess. Manag. 2019, 15, 312.

[18] J. Bengtsson-Palme, D. G. J. Larsson, Environ. Int. 2016, 86, 140.

[19] J. O. Straub, Antibiotics 2013, 2, 115.

[20] J. O. Straub, Environ. Toxicol. Chem. 2015, 35, 767.

[21] D. J. Caldwell, B. Mertens, K. Kappler, T. Senac, R. Journel, P. Wilson, R. D. Meyerhoff, N. J. Parke, F. Mastrocco, B. Mattson, R. Murray-Smith, D. G. Dolan, J. O. Straub, M. Wiedemann, A. Hartmann, D. S. Finan, Environ. Toxicol. Chem. 2016, 35, 813. 
[22] R. Loos, D. Marinov, I. Sanseverino, D. Napierska, T. Lettieri, 'Review of the $1^{\text {st }}$ Watch List under the Water Framework Directive and recommendations for the $2^{\text {nd }}$ Watch List', Publications Office of the European Union, Luxembourg, 2018, ISBN 978-92-79-81839-4, cited July 24, 2019, available from ec.europa.eu/jrc/en/publication/review-1st-watch-list-under-water-framework-directive-and-recommendations-2nd-watch-list JRC111198

[23] J. Vestel, D. J. Caldwell, L. Constantine, V. J. D’Aco, T. Davidson, D. G. Dolan, S. P. Millard, R. Murray-Smith, N. J. Parke, J. J. Ryan, J. O. Straub P. Wilson, Environ. Toxicol. Chem. 2016, 35, 1201.

[24] New Pharma PNEC List 9.11.18, updated September 2018, WET Center, Temple University, Philadelphia, PA, USA, cited July 11, 2019, available from www.nsfwetcenter.org/wp-content/uploads/2018/09/WET-CenterPhamaceutical-PNEC-list-09102018-1.xlsx

[25] FASS online database, cited July 5, 2019, available from www.fass.se/LIF/ startpage

[26] GlaxoSmithKline Safety Data Sheets, cited July 5, 2019, available from www.msds-gsk.com/(S(fnptzeqpqplpvd55li40qv55))/SDSList.aspx

[27] F.Hoffmann-La Roche Safety Data Sheets, cited July 5, 2019, available from www.roche.com/sustainability/environment/safety data_sheets-row.htm

[28] AstraZeneca plc, Abbreviated Environmental Risk Assessments, cited July 5, 2019, available from www.astrazeneca.com/content/dam/az/PDF/2017/ Environmental risk_data_relating to our medicines.pdf

[29] GlaxoSmithKline plc, Abbreviated Environmental Risk Assessments, cited July 5, 2019, available from www.msds-gsk.com/ (S(fnptzeqpqplpvd55li40qv55))/ERAList.aspx
[30] F.Hoffmann-La Roche Ltd, Abbreviated Environmental Risk Assessments, cited July 5, 2019, available from www.roche.com/sustainability/environment/environmental-risk-assessment-downloads.htm

[31] 'Green Chemical Syntheses and Processes', Eds. P. T. Anastas, L. G. Heine, T. C. Williamson, ACS Symposium Series Vol. 767, American Chemical Society, Washington, DC 20036, USA, 2000.

[32] P. J. Dunn, S. Galvin, K. Hettenbach, Green Chem. 2004, 6, 43.

[33] W. J. W. Watson, Green Chem. 2012, 14, 251.

[34] B. W. Cue, J. Zhang, Green Chem. Lett. Rev. 2009, 2, 193.

[35] H. Sugiyama, R. Schmidt, Comput-Aided Chem. Eng. 2013, 32, 697.

\section{License and Terms}

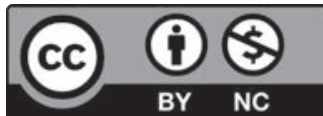

This is an Open Access article under the terms of the Creative Commons Attribution License CC BY_NC 4.0. The material may not be used for commercial purposes.

The license is subject to the CHIMIA terms and conditions: (http:// chimia.ch/component/sppagebuilder/?view = page \&id=12).

The definitive version of this article is the electronic one that can be found at doi:10.2533/chimia.2020.161 